\title{
Utility of Nontraditional Data Sources for Early Detection of Influenza
}

\author{
Shari Barlow ${ }^{2}$, Jonathan L. Temte ${ }^{2}$, Yenlik Zheteyeva*1, Ashley Fowlkes ${ }^{3}$, Carrie Reed ${ }^{3}$ \\ and Derek Cummings ${ }^{4}$
}

${ }^{1} \mathrm{DGMQ}, \mathrm{CDC}$, Atlanta, GA, USA; ${ }^{2}$ Department of Family Medicine and Community Health, University of Wisconsin, Madison, WI, USA; ${ }^{3}$ Influenza Division, CDC, Atlanta, GA, USA; ${ }^{4}$ University of Florida, Department of Biology, Emerging Pathogens Institute,

Gainesville, FL, USA

\section{Objective}

This session will provide an overview of the current systems for influenza surveillance; review the role of schools in influenza transmission; discuss relationships between school closures, school absenteeism, and influenza transmission; and explore the usefulness of school absenteeism and unplanned school closure monitoring for early detection of influenza in schools and broader communities.

\section{Introduction}

Influenza surveillance is conducted through a complex network of laboratory and epidemiologic systems essential for estimating population burden of disease, selecting influenza vaccine viruses, and detecting novel influenza viruses with pandemic potential (1). Influenza surveillance faces numerous challenges, such as constantly changing influenza viruses, substantial variability in the number of affected people and the severity of disease, nonspecific symptoms, and need for laboratory testing to confirm diagnosis. Exploring additional components that provide morbidity information may enhance current influenza surveillance.

School-aged children have the highest influenza incidence rates among all age groups. Due to the close interaction of children in schools and subsequent introduction of influenza into households, it is recognized that schools can serve as amplification points of influenza transmission in communities. For this reason, pandemic preparedness recommendations include possible pre-emptive school closures, before transmission is widespread within a school system or broader community, to slow influenza transmission until appropriate vaccines become available. During seasonal influenza epidemics, school closures are usually reactive, implemented in response to high absenteeism of students and staff after the disease is already widespread in the community. Reactive closures are often too late to reduce influenza transmission and are ineffective.

To enhance timely influenza detection, a variety of nontraditional data sources have been explored. School absenteeism was suggested by several research groups to improve school-based influenza surveillance. A study conducted in Japan demonstrated that influenzaassociated absenteeism can predict influenza outbreaks with high sensitivity and specificity (2). Another study found the use of allcauses absenteeism to be too nonspecific for utility in influenza surveillance (3). Creation of school-based early warning systems for pandemic influenza remains an interest, and further studies are needed. The panel will discuss how school-based surveillance can complement existing influenza surveillance systems.

\section{Keywords}

Surveillance; Absenteeism; Influenza; Early Detection; Schools

\section{References}

Brammer L, Budd A, Cox N. Seasonal and pandemic influenza surveillance considerations for constructing multicomponent systems. Influenza Other Respi Viruses. 2009;3:51-58.
Sasaki A, Hoen AG, Ozonoff A, et al. Evidence-based tool for triggering school closures during influenza outbreaks, Japan. EID. 2009;15: 1841-1843.

Besculides M, Heffernan R, Mostashari F, et al. Evaluation of school absenteeism data for early outbreak detection, New York City. BMC PH. 2005;5:105.

\section{*Yenlik Zheteyeva}

E-mail: igg0@cdc.gov 